\title{
An Improved Method for Viable Counts of Bacteria of the Ovine Rumen which ferment Carbohydrates
}

\author{
By A. KISTNER* \\ National Chemical Research Laboratory, South African Council for Scientific and \\ Industrial Research, Pretoria, South Africa
}

(Received 2 June 1960)

\begin{abstract}
SUMMARY
A method is described which is suitable for routine viable counts of rumen bacteria capable of fermenting various carbohydrates. The contribution of different steps in the preparation of the medium towards the final redox potential was determined and the choice of a suitable reducing agent, redox indicator and source of rumen fluid supplement is discussed. In a sheep on a constant practical ration, the variation in numbers of cellulolytic bacteria was greater than the differences between five successive samples withdrawn at one time, and much greater than the variation due to the method.
\end{abstract}

\section{INTRODUCTION}

There has been considerable interest in recent years in the role played by the bacterial population of the rumen in the digestive processes of the host animal. These rumen bacteria may be divided into three groups: (1) functional bacteria; (2) bacteria accidentally introduced with food and water; (3) potentially harmful bacteria normally present in insignificant numbers (Gilchrist \& Clark, 1957). In studies on the influence of the ruminal flora on the metabolism of the ruminant, the main interest lies with the first-mentioned group, and several authors have proposed sets of criteria for establishing whether a bacterial strain is a functional member of the rumen population or not (Gall \& Huhtanen, 1951; Briggs, 1955; Bryant, 1959). These criteria are all elaborations on the two fundamental principles laid down by Elsden \& Phillipson (1948), namely, that a functional ruminal organism is one which performs a chemical reaction known to occur in the rumen and is present in numbers sufficient to perform this reaction at a rate comparable with that occurring in the rumen. One of the first requirements in the study of functional rumen bacteria is thus the ability to determine the number of bacteria capable of bringing about a certain biochemical reaction.

Although the importance of this type of determination was recognized more than a decade ago, very few quantitative studies have been made. One of the reasons for this is that there is no satisfactory routine method for performing counts of this nature. It is now generally recognized that many functional rumen bacteria are closely adapted to their peculiar environment and cannot be cultivated by conventional bacteriological methods. To obtain valid viable counts of the different metabolic groups of rumen bacteria, it is, therefore, essential to provide cultural

* Postal address : c/o The Director of Veterinary Services, P.O. Onderstepoort, Pretoria. 
conditions which closely resemble those existing in the rumen of the experimental animal. The most important conditions are : (1) the average temperature of the rumen $\left(c .38 .5^{\circ}\right)$ as the temperature of incubation; (2) an inorganic salt composition of the medium similar to that of the ruminant's saliva; (3) bicarbonate + carbon dioxide as the main buffer system of the medium; (4) a certain percentage of rumen fluid as a source of growth factors and reducing agents; (5) the maintenance of a low redox potential in the medium. The importance of some of these factors in the cultivation of functional rumen bacteria was discussed in detail by Bryant (1959).

Bryant \& Burkey $(1953 a, b)$ described a method which took into account the above-mentioned cultural requirements and which enabled them to determine the number of bacteria capable of fermenting a variety of carbohydrate substrates. However, the method involves the quantitative isolation of the colonies obtained by inoculation of high dilutions of rumen contents on a non-selective solid medium and subsequent determination of the fermentation reactions of the resultant cultures and is therefore much too cumbersome for routine determinations of the composition of the bacterial population of the rumen under different dietary conditions. In view of this, it was decided to follow a more direct method by counting the number of colonies which developed on selective solid media containing single carbohydrate substrates. A certain amount of overlapping of the counts on the different media was to be expected, but this would not be too serious as the results were not intended for estimation of total counts. The medium and techniques of Hungate (1950) were selected as being the most suitable as the basis of the proposed method. However, these were intended for isolation studies and were not convenient for routine counts. They were therefore modified to give greater speed and accuracy. At the same time, more rigorous anaerobic precautions and a more sensitive redox indicator were introduced to ensure consistent growth of some of the predominant types of cellulolytic bacteria. The present communication describes the method finally evolved as well as the rationale of the various steps in the procedure and the reproducibility and limitations of the method.

\section{METHODS}

\section{Determination of oxidation-reduction potentials in media}

The potentials were measured with a Metrohm 280-E battery-operated $\mathrm{pH}$ and millivolt meter which has an accuracy of $\pm 10 \mathrm{mV}$. The electrode system was: unknown solution $\mathrm{Pt} / \mathrm{KCl}$ bridge/ $\mathrm{Ag} / \mathrm{AgCl}$, saturated $\mathrm{KCl}$. The platinum and reference electrodes were combined in a single probe provided with a standard groundglass joint. The conventional method of cleaning platinum electrodes by immersion in hot strong nitric acid followed by rinsing with distilled water resulted in a very slow equilibration of the electrode with strongly reducing systems. This situation was remedied by subjecting the platinum surface to an electrolytic treatment immediately before use. The electrode was immersed in $100 \mathrm{ml}$. N-HCl solution containing $\mathrm{l}$ drop of Teepol and connected to the negative pole of a 6-V. dry battery. A graphite rod served as anode and the electrolysis was continued for 3 min., after which the electrode was rinsed with distilled water and immediately fitted into the lid of a Metrohm 2-part titration vessel which had previously been purged with oxygenfree $\mathrm{CO}_{2}$. The medium of unknown $E_{h}$ value was introduced into the titration vessel 
under strictly anaerobic precautions and was stirred by means of a current of oxygenfree $\mathrm{CO}_{2}$. The vessel was also fitted with a combined glass and reference electrode so that $E_{h}$ and $\mathrm{pH}$ values could be recorded simultaneously.

\section{Method for making viable counts}

The methods finally adopted were as follows:

Gassing. Air was displaced from solutions, media and culture vessels by gassing with sterile oxygen-free $\mathrm{CO}_{2}$ or, where the high solubility of this gas in aqueous solutions was objectionable, with $\mathrm{N}_{2}$. The commercial gases were freed from $\mathrm{O}_{2}$ by passing them over palladinized copper turnings held at $300^{\circ}$. With the aid of a manifold and flowmeters, six equal currents of oxygen-free gas could be obtained, which were rendered germ-free by passing through sterilized filters. These consisted of the barrels of $2 \mathrm{ml}$. Luer Lock syringes stuffed with cotton wool. For gassing the smaller vessels, these filters were provided with 19-gauge hypodermic needles of sufficient length to reach the bottoms of the vessels. Rubber-stoppered bottles were gassed by inserting the needle between the rubber stopper and the neck of the bottle. They were sealed without entry of air by carefully withdrawing the needle with the right hand while holding down the stopper with the left thumb and forefinger. The rate of gassing was usually approximately $12 \mathrm{l} . / \mathrm{hr}$.

Substrates. The final concentrations of the substrates employed in this study were: soluble starch (Merck's 'pro analysi ') $1 \cdot 2 \%$ (w/v); glucose (Merck's 'for bacteriology') $1.0 \%(w / v) ; d(+)$ xylose (Merck's 'pure chemicals') $1.0 \%(w / v) ;$ a finely ground suspension of acid-treated cellulose prepared according to Hungate (1950) $1 \cdot 2 \%$ $(\mathrm{w} / \mathrm{v})$.

Rumen fluid supplement. Rumen ingesta were withdrawn by suction tube from a fistulated sheep before morning feeding. As a rule the rumen fluid was obtained from animals on a lucerne hay diet (see below). The ingesta were strained through a double thickness of cheesecloth and the fluid collected in a 1-pint plastic bottle which was completely filled. The bottle was sealed with a rubber bung and chilled in crushed ice for at least $\mathbf{3 0} \mathrm{min}$. to bring gaseous fermentation to a standstill. In order to keep the coarser particles of the rumen contents out of the medium where they might later be confused with small colonies, the bottle was centrifuged at $1200 \mathrm{~g}$ for $20 \mathrm{~min}$. When required, the supernatant fluid was carefully removed by means of a $50 \mathrm{ml}$. syringe with a $4 \mathrm{in}$. cannula while the gas space above the fluid was purged with oxygen-free $\mathrm{CO}_{2}$ to prevent absorption of atmospheric $\mathrm{O}_{2}$.

The composition of the medium was as follows: $\left(\mathrm{NH}_{4}\right)_{2} \mathrm{SO}_{4}, 5 \%$ (w/v) stock solution, $5 \mathrm{ml}$.; $\mathrm{K}_{2} \mathrm{HPO}_{4}, 5 \%$ (w/v), $5 \mathrm{ml}$.; $\mathrm{KH}_{2} \mathrm{PO}_{4}, 2 \%$ (w/v), $5 \mathrm{ml}$.; $\mathrm{CaCl}_{2}, 0.5 \%$ (w/v), $5 \mathrm{ml}$; $\mathrm{MgSO}_{4} .7 \mathrm{H}_{2} \mathrm{O}, 0.5 \%$ (w/v), $5 \mathrm{ml}$; $\mathrm{NaCl}, 10 \%(\mathrm{w} / \mathrm{v}), 5 \mathrm{ml}$.; Antifoam $\mathrm{B}$ (Dow Corning), $10 \%(\mathrm{v} / \mathrm{v})$ emulsion in distilled water, $5 \mathrm{ml}$; indigodisulphonate, $0.05 \%$ stock solution, $5 \mathrm{ml}$; cysteine $\mathrm{HCl}, 250 \mathrm{mg}$.; $\mathrm{NaHCO}_{3}, 2.6$ g.; processed rumen fluid, $150 \mathrm{ml}$; solution or suspension of the substrate to give the final concentration stated above; deionized water to $500 \mathrm{ml}$.

Cellulose and starch were sterilized together with the basal medium; glucose and xylose were added to the molten agar after sterilization in the form of $25 \%$ $(\mathrm{w} / \mathrm{v})$ Seitz-filtered stock solutions.

Preparation of medium. Agar (7.5 g.) was weighed into a $40 \mathrm{oz}$. screw-capped bottle, the appropriate volume of distilled water was added and the bottle heated 
in a boiling water-bath until the agar was dissolved. When cellulose or starch was to be substrate, $150 \mathrm{ml}$. of a hot $4 \%(\mathrm{w} / \mathrm{v})$ suspension or solution were added to the molten agar at this stage, followed by the above-mentioned quantities of inorganic salt solutions, redox indicator and anti-foaming agent. Oxygen-free $\mathrm{CO}_{2}$ was then bubbled through the hot mixture by means of a 16-gauge stainless-steel cannula inserted between the neck of the bottle and a well-fitting rubber stopper. The rate of bubbling was fast enough to produce turbulence in the medium.

After gassing for a minimum of $5 \mathrm{~min}$., the bottle was removed from the waterbath and cysteine. $\mathrm{HCl}$ added to the medium, followed by $150 \mathrm{ml}$. of centrifuged rumen fluid. The medium was now cooled to $c .47^{\circ}$ before the $\mathrm{NaHCO}_{3}$ was added. After replacing the rubber stopper, gassing was continued for another $10 \mathrm{~min}$. to

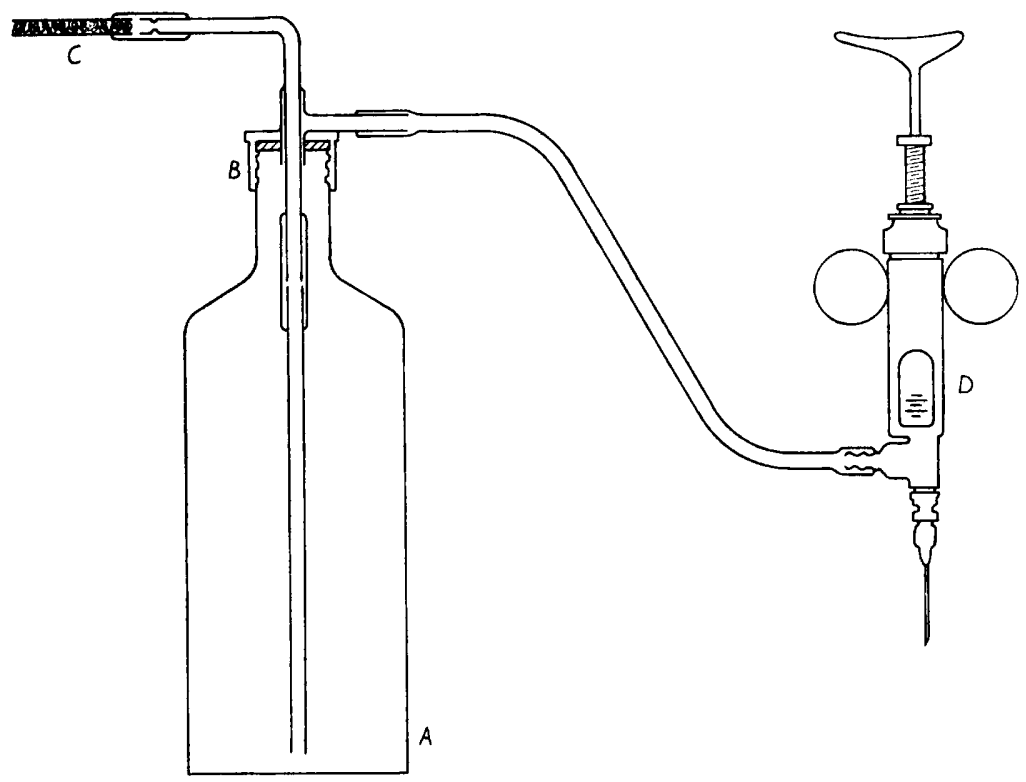

Fig. 1. Automatic pipetting outfit. $A, 40 \mathrm{oz}$. McCartney bottle (United Glass Bottle Manufacturers, London); $B$, filterhead (Gallenkamp, London) with inlet tube extended to the bottom of the bottle by means of glass tubing; $C$, cotton-wool filter; $D$, automatic syringe, $5 \mathrm{ml}$. capacity (Milborrow and Co., Pietermaritzburg), connected to $B$ by means of latex tubing.

saturate the medium with $\mathrm{CO}_{2}$. The cannula was then carefully withdrawn to prevent re-entry of air, and the rubber stopper sealed down by a screw cap with paper liner. The medium was immediately sterilized at $120^{\circ}$ for $25 \mathrm{~min}$. and then allowed to cool to $47^{\circ}$ before distribution.

Where glucose or xylose was to be the substrate, $20 \mathrm{ml}$. of a $25 \%$ (w/v) Seitzfiltered stock solution were injected into the bottle, after sterilization, through the rubber stopper, and thoroughly mixed with the medium by shaking.

Distribution of medium over dilution bottles. Moist $1 \mathrm{oz}$. screw-capped bottles were provided with well-fitting rubber stoppers and flushed out with oxygen-free $\mathbf{N}_{2}$ for 5 min. as described under 'Gassing' above. The screw caps were then fitted over the rubber stoppers and the bottles sterilized at $120^{\circ}$ for $30 \mathrm{~min}$., together with the automatic pipetting outfit shown in Fig. 1. After cooling to room temperature, the 
pipetting outfit was connected to the gas manifold and, with $\mathrm{CO}_{2}$ passing through the inlet tube, the empty bottle was quickly replaced by a bottle of sterile molten agar medium at $47^{\circ}$, with aseptic precautions. The filter head was left slightly loose to allow gas to escape between the rim of the bottle and the filter head, and the bottle was purged with oxygen-free $\mathrm{CO}_{2}$ for another $5 \mathrm{~min}$. After tightening the filter head, air was displaced from the rubber tubing and the syringe by depressing and releasing the plunger of the pipetting outfit 20 times. By inverting the bottle and working the plunger, the medium was alternately fed into the syringe and injected into the dilution bottles in $9.0 \mathrm{ml}$. quantities through the rubber stoppers. The bottles were stored at room temperature. When required, the medium was melted and the solid screw caps were replaced by sterile caps with two $\frac{1}{16}$ in. perforations $c . \frac{1}{2}$ in. apart.

Modified cellulose medium. Sodium dithionite was substituted for cysteine in the cellulose agar to give a final concentration of $0.003 \%(w / v)$. As dithionite is decomposed by heat, $0.2 \mathrm{ml}$. of a freshly prepared $0.15 \%$ solution in oxygen-free water under $\mathrm{N}_{2}$ pressure was added to $8.8 \mathrm{ml}$. molten agar in the dilution bottles shortly before use. The solution could be conveniently sterilized by passing it through a Swinny filter adapter (Becton, Dickinson and Co., Rutherford, N.J.) containing a $\frac{1}{2}$ in. Seitz filter mat.

Sample dilutions. The samples of rumen ingesta were taken by means of a suction tube from fistulated animals. The ingesta were strained through a double thickness of cheesecloth and the liquor collected in a glass-stoppered bottle which was completely filled and immediately returned to the laboratory. By means of a Luer Lock type syringe with a 20 -gauge $\times 1 \frac{1}{4}$ in. needle, $1 \mathrm{ml}$. of sample was injected into a dilution bottle containing $9 \mathrm{ml}$. of molten agar medium at $47^{\circ}$; thorough mixing was obtained by rolling the bottle between the hands and simultaneously turning the wrists through an angle of $180^{\circ}$. With a fresh sterile syringe $1 \mathrm{ml}$. of the first dilution was then withdrawn through a second perforation of the rubber stopper -to avoid contamination of the needle with a trace of the original sample-and injected into the next bottle. In this way a series of tenfold dilutions was prepared, the degree of dilution required to obtain a countable number of colonies in the roll bottles depending on the substrate used and the composition of the ruminal flora of the particular animal.

Beginning with the highest dilution, $4 \mathrm{ml}$. of each of the last four dilutions was drawn into a Luer Lock syringe and dispensed over four sterile $\frac{1}{4}$ oz. rubber-stoppered roll bottles which had previously been filled with $\mathrm{CO}_{2}$. In order to remove any $\mathrm{O}_{2}$ which might have been introduced during this process, the bottles were immediately purged with oxygen-free $\mathrm{CO}_{2}$ for 2 min., while partially immersed in the $47^{\circ}$ waterbath. The bottles were then spun, four at a time, in an indirectly water-cooled solidification apparatus (Apparatenfabriek van Dooren, de Bilt, Netherlands) for $3 \mathrm{~min}$. to obtain a uniform film of agar of $c .2 \mathrm{~mm}$. thickness along the walls of the roll bottle. The bottles were incubated upside-down to allow the water of syneresis to drain off.

Incubation times at $38 \cdot 5^{\circ}$. Starch medium $16 \mathrm{hr}$.; glucose and xylose media $20 \mathrm{hr}$.; cellulose medium 5-42 days depending on the rate of growth of the cellulolytic bacteria in the sample.

Counting. The counting device of Apparatenfabriek van Dooren was used; this 
permits scanning of the surface of the bottle in spiral fashion. The bottles were illuminated from below and the colonies were viewed at a magnification of $\times 10$. Whenever the redox indicator showed that a bottle had absorbed $\mathrm{O}_{2}$, the colony count was disregarded in calculating the average number for that particular dilution. On cellulose media only those colonies which were surrounded by a visible zone of clearing were counted. Similarly, the colony counts on starch media were made specific by spreading a few drops of Lugol's iodine solution over the agar and counting only those colonies which were surrounded by a concentric colourless zone.

\section{RESULTS AND DISCUSSION}

\section{Redox potential of the medium}

Although it is now generally recognized that the maintenance of a low redox potential in the medium is of utmost importance in the cultivation of predominant rumen bacteria, few data have been published on the actual $\boldsymbol{E}_{h}$ values obtained in the media by the use of different reducing agents, as compared with the potentials prevailing in the rumen of the experimental animal from which the samples were to be taken for cultivation. Huhtanen, Rogers \& Gall (1952) used cysteine as reducing agent in their sample-diluting fluid and in their medium. In the former, they obtained a redox potential of $-200 \mathrm{mV}$. at $\mathrm{pH} 6.8$ which is well above the $E_{h}$ value which they reported for the rumen contents of cattle on a high roughage ration, namely, $-300 \mathrm{mV}$. (Huhtanen \& Gall, 1953). Nevertheless, these authors succeeded in obtaining growth of several different types of rumen bacteria from high dilutions of the sample. On the other hand, Smith \& Hungate (1958) found that they could not cultivate the methane bacteria from the rumen of cattle unless the redox potential of the medium was low enough to reduce benzylviologen $\left(E_{0}^{\prime}-359 \mathrm{mV}\right.$. at $\left.\mathrm{pH} \mathbf{7 \cdot 0}\right)$ which was included as indicator. The $E_{h}$ value of the rumen contents which served as the inoculum for their isolations varied between -335 and $-346 \mathrm{mV}$.

In the early stages of the present investigation Hungate's (1950) basal medium containing $0.01 \%(\mathrm{w} / \mathrm{v})$ cysteine hydrochloride was used. On this medium consistent counts were obtained of the groups of bacteria which fermented starch, glucose and xylose and also of the rod-shaped cellulolytic bacteria predominating in rumen contents of sheep on teff hay. When this type of medium was inoculated with rumen fluid from sheep fed lucerne hay, in which cocci appear to be the predominant cellulolytic organisms, rapid growth and complete digestion of the cellulose was usually observed in the first dilution. However, instead of the expected tenfold decrease in the number of colonies from one dilution to the next, there was an abrupt break at the second or third dilution and no growth whatsoever beyond that. The possibility existed that this phenomenon was due to a heat-labile growth factor normally present in rumen fluid which was destroyed by heat sterilization of the medium, then reintroduced with the inoculum only to be diluted out with progressive tenfold dilutions. However, this explanation was ruled out by the fact that good growth of the same bacteria was obtained even in the high dilutions when cellobiose was substituted for cellulose. This observation suggested another possibility, namely, that the initiation of the growth of the cocci on media containing a refractory substrate such as cellulose required lower redox potential values than on readily available carbohydrates such as cellobiose. As a first step in testing this hypothesis, 
the contribution of the different steps in the preparation of the Hungate type medium to the final $E_{h}$ value was examined. Table 1 shows a typical set of measurements.

From these measurements it appears that the redox potential of the medium was determined by the rumen fluid supplement and that the contribution of the cysteine would be an improved poising capacity rather than a lower $E_{h}$ value. It may also be seen that the $E_{h}$ of the medium rose during the sterilization process to approximately that obtained by the addition of cysteine, with the result that the final potential was considerably higher than that of the rumen contents of the animal from which the sample was taken.

In the course of these measurements it was discovered that the method of removing oxygen from commercial gases by passing these through wash bottles containing a chromous acid solution, as recommended by Hungate (1950), was not effective in the case of our bottled carbon dioxide. When this gas was used to stir the medium in the titration vessel during $E_{h}$ measurements, the redox potential became progressively more positive, while this tendency ceased as soon as the gas flow was interrupted. A furnace containing palladinized copper turnings at $\mathbf{3 0 0}^{\circ}$

Table 1. The contribution of different steps in the preparation of the Hungate-type medium to the final $\boldsymbol{E}_{h}$

$$
\text { Step }
$$

(1) $70 \mathrm{ml}$. boiled out basal medium in equilibrium with oxygen-free $\mathrm{CO}_{2}$

(2) $10 \mathrm{mg}$. cysteine. $\mathrm{HCl}$ added

(3) Clarified rumen fluid supplement from a sheep fed lucerne hay

(4) $30 \mathrm{ml}$. clarified rumen fluid added to basal medium

(5) Medium after sterilization at $120^{\circ}$ for $20 \mathrm{~min}$.

$\begin{array}{cr}\begin{array}{c}\mathrm{pH} \\ \text { value }\end{array} & \begin{array}{c}E_{h} \\ (\mathrm{mV} .)\end{array} \\ 6 \cdot 8 & +55 \\ 6 \cdot 8 & -210 \\ - & -300 \\ 6 \cdot 8 & -271 \\ 6.7 & -160\end{array}$

was therefore substituted for the chromous acid absorption train and, when the effluent gas from this set-up was passed through a weakly poised medium of low redox potential, no change in potential occurred within $15 \mathrm{~min}$., thus indicating that the residual oxygen content was very low.

Choice of reducing agent. Inspection of the $E_{0}^{\prime}$ values of such biological reducing agents as glutathione, ascorbic acid and thioglycollate indicated that they could not be expected to be superior to cysteine in the production of low redox potentials in our media. However, the novel biological method used by Smith \& Hungate (1958) to obtain good poising capacities at low $E_{h}$ values by growing Escherichia coli in the medium and subsequently killing this organism by a pasteurization process without loss of reducing power, looked promising. Unfortunately, this method did not work at our hands as the strain of $E$. coli used would not grow in our medium. However, another method suggested by Smith \& Hungate was applied with better success, namely, the use of $0.003 \%(\mathrm{w} / \mathrm{v})$ sodium dithionite. It was pointed out by these authors that the low concentration of dithionite tolerated by their rumen methane bacteria resulted in a poorly poised medium which tended to give erratic growth. Nevertheless, we found that the low potentials imparted by dithionite to the medium ( -400 to $-440 \mathrm{mV}$. at $\mathrm{pH} 6 \cdot 8$ ) could be maintained throughout the 
procedures of our techniques provided that sufficiently strict anaerobic precautions were taken. Owing to its heat lability, the dithionite had to be added in the form of a freshly prepared Seitz-filtered solution to the molten agar medium immediately before inoculation.

On the dithionite-containing media, good counts of the cellulolytic cocci from sheep fed on lucerne hay were obtained. It was found that these cocci would also grow on cellulose media without added dithionite, provided that the carbon dioxide used for gassing the medium and the cultures had a very low oxygen content. However, the growth of these organisms was more consistent in the presence of dithionite. The use of the modified cellulose medium containing dithionite was, therefore, adopted as a standard procedure for counting all types of cellulolytic bacteria. In the case of the functional rumen bacteria which fermented starch, glucose and xylose, no advantage was gained by substituting dithionite for cysteine in the medium. Moreover, the accurate dosage of dithionite solution into the medium immediately before inoculation involved a considerable amount of labour; hence it was decided to revert to the use of cysteine in the media for these groups of bacteria.

Choice of redox indicator. From the figures quoted above for the redox potential of Hungate type media, it would appear that the indicator which has been most widely used in media for rumen bacteria, namely resazurine, is not well suited for the purpose. The normal potential of this indicator at $\mathrm{pH} 7 \cdot 0$ is $+51 \mathrm{mV}$., and it will show its oxidized colour only after most of the cysteine in the medium has been oxidized. This might explain the observations of Bryant (1959) that the growth of Bacteroides succinogenes in a chemically defined medium was erratic as long as he relied on resazurine as an indicator of a suitably low redox potential in the medium. Only when he introduced more stringent anaerobic precautions, including the use of the more sensitive indicator indigodisulphonate $\left(E_{0}^{\prime}-125 \mathrm{mV}\right.$. at $\left.\mathrm{pH} 7 \cdot 0\right)$, did he obtain consistent growth of this organism. The suitability of indigodisulphonate as a redox indicator in different cysteine-containing media for rumen bacteria was tested in the present work. The concentration recommended by Bryant $(0.0005 \%)$ produced a visible colour depth of the oxidized form even in the thin layer of agar in the roll bottles, and no toxic effect has thus far been detected on the organisms developing on the different media. However, when tubes of an old batch of medium were exposed to the air, only a faint colour was produced; this limited the usefulness of the indicator in the preparation of large batches of medium to be stored for routine work.

For counts on the modified cellulose medium containing $0.003 \%$ sodium dithionite, the use of an even more sensitive redox indicator seemed appropriate. Inspection of tables on redox indicators suggested that benzylviologen $\left(E_{0}^{\prime}-359 \mathrm{mV}\right.$. at $\mathrm{pH} \mathrm{7 \cdot 0)}$ ) should be ideally suited for the purpose. In fact, this indicator was used with good success by Smith \& Hungate (1958) in media for their rumen methane bacteria. However, it was found that the concentration used by these authors, namely, $\mathbf{0 . 0 0 1} \%$, tended to produce low counts on media containing glucose. Furthermore, this concentration of the indicator did not produce a visible depth of colour in the thin film of cellulose medium in the roll bottles and when the concentration was increased to $0.005 \%$, growth of the cellulolytic cocci was almost completely inhibited. Resort was made to indigodisulphonate (indigocarmine) added to the medium together with the dithionite solution immediately before inoculation. 


\section{Choice of rumen fluid supplement}

Initially, counts were made on media containing rumen fluid from sheep on the same diet as the animal whose rumen flora was under examination. This was done to ensure that the growth factor requirements of the functional bacteria predominating under those dietary conditions would be satisfied. However, the application of this principle proved to be very tedious in practice since a complete set of media had to be prepared for every diet studied. It was decided therefore to test the feasibility of standardizing the medium by using rumen fluid from sheep fed a diet of lucerne hay on which the animal could be kept indefinitely. For the metabolic groups of rumen bacteria which ferment starch, glucose and xylose, and also for the cellulose digesters from teff hay-fed sheep, there was little difference whether the rumen fluid was derived from an animal fed teff or lucerne hay. A difference was found in the case of the cellulolytic cocci from sheep on lucerne hay: these grew faster and more abundantly on media supplemented with rumen fluid from animals on lucerne hay. It was therefore considered advantageous to standardize on rumen fluid from this source. In order to avoid introduction of appreciable quantities of extraneous growth substrates in the medium, the rumen fluid sample was withdrawn from the animals before morning feeding.

\section{Sample treatment}

It is realized that the method of preparing the sample of rumen contents by straining it through cheesecloth eliminates the 'fixed' bacteria, that is, those attached to the larger food particles. This leads to low counts of organisms such as the cellulolytic bacteria, a proportion of which is known to be located in cavities of the partially digested fibres. At present there is no method for removing these organisms quantitatively from the food particles without danger of impairing their viability, and any method which achieves only partial liberation of the fixed bacteria may be expected to result in a variation of counts which is no true reflexion of the changes occurring in the rumen. On the other hand, there is good reason to believe that the proportion of fixed to free organisms will be reasonably constant at a standard time after feeding. Since the method presented was intended for comparative counts of certain functional groups of rumen bacteria under different dietary conditions, rather than for obtaining absolute values for each group, the present system of sample treatment was chosen as the best practical compromise.

\section{Tests of the method}

Reproducibility of counts on a single sample. This was determined by running five parallel dilution series on cellulose agar, using a well-mixed sample of strained rumen contents from a lucerne hay-fed sheep as inoculum. Cellulose agar was chosen because it has been found that of the carbohydrate-fermenting functional rumen bacteria the cellulose digesters were the most difficult to cultivate and could, therefore, be expected to show the greatest variation in numbers. The sample was distributed over five $1 \mathrm{oz}$. screw-capped bottles which were completely filled and immediately chilled in ice in order to prevent changes in the composition of the bacterial population in the time interval between the withdrawal and the processing of the sample. For the same reason the subsamples were processed by a team of 
four workers, the operations of dilution, mixing, distribution and gassing being repeated over and over by the same workers to minimize the effect of personal errors. In this way, the dilution series from the last subsample was ready for incubation within $75 \mathrm{~min}$. after collection of the sample.

The colony counts after 14 days of incubation of the four roll bottles of the sixth and seventh dilutions of each subsample are shown in Table 2. In this experiment there were no colonies in the sixth dilution of the second subsample, although there was good growth in the corresponding fifth and seventh dilutions. This is a very rare occurrence and may have been caused by contamination of that particular bottle with a toxic substance. The decrease of numbers of colonies from the sixth to the seventh dilution is in good agreement with the expected tenfold decrease. Moreover, considering that the growth of the cellulolytic rumen bacteria in vitro is very much dependent on the provision of their special environmental requirements in the medium, the coefficient of variation of the results obtained by our method, namely, $3.95 \%$,

Table 2. Replicate counts of cellulolytic bacteria in a sample of rumen contents from a sheep fed lucerne hay

\begin{tabular}{|c|c|c|c|c|c|c|c|c|c|}
\hline \multicolumn{5}{|c|}{ Subsamples: dilution $1 / 10^{6}$} & \multicolumn{5}{|c|}{ Subsamples: dilution $1 / 10^{7}$} \\
\hline 1 & 2 & 3 & 4 & 5 & 1 & 2 & 3 & 4 & 5 \\
\hline 20 & $\mathbf{0}$ & 21 & 23 & 16 & 2 & 1 & 3 & $\mathbf{3}$ & 3 \\
\hline 23 & $\mathbf{0}$ & 27 & 21 & 16 & $\mathbf{0}$ & 3 & 3 & $\mathbf{0}$ & 1 \\
\hline 20 & $\mathbf{0}$ & 15 & 20 & 19 & 1 & $\mathbf{3}$ & 2 & 1 & 3 \\
\hline 23 & $\mathbf{0}$ & 19 & 19 & 26 & $\mathbf{2}$ & 4 & 2 & 1 & 1 \\
\hline \multirow[t]{2}{*}{$21 \cdot 5$} & $\mathbf{0}$ & $20 \cdot 5$ & $20 \cdot 75$ & $19 \cdot 25$ & $1 \cdot 25$ & $2 \cdot 75$ & $\mathbf{2 \cdot 5}$ & $\mathbf{1} \cdot \mathbf{2 5}$ & $2 \cdot 0$ \\
\hline & \multicolumn{6}{|c|}{$\begin{array}{l}\text { Mean of } 4 \text { determinations at } 1 / 10^{6} \text { dilution } \\
\text { Standard deviation } \\
\text { Coefficient of variation between subsamples }\end{array}$} & \multicolumn{3}{|c|}{$\begin{array}{l}20.5 \\
0.81 \\
\mathbf{3 . 9 5} \%\end{array}$} \\
\hline
\end{tabular}

compares very favourably with the $7 \cdot 15 \%$ reported by Cook \& Steel (1959) for dilution series of an Escherichia coli suspension prepared by their second method, which corresponds to ours.

Sampling error. In view of the fact that the rumen ingesta are not homogeneous (Pearson \& Smith, 1943) and that it is difficult to withdraw repeat samples from exactly the same part of the rumen, it was considered necessary to determine also the magnitude of the variations in counts due to the sampling technique. A knowledge of the sampling error is of importance in the interpretation of the results of comparative counts run on different days. The plan of the experiment was similar to that of the previous one with the exception that five consecutive small samples were withdrawn from random positions within the rumen and were separately strained and collected in 1 oz. screw-capped bottles. The counts of cellulolytic colonies in the sixth dilution after 14 days of incubation are shown in Table 3. From these results it appears that the coefficient of variation between consecutive samples,

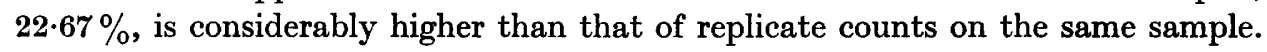
It is, however, of the same order as that found for differences in the chemical analyses of samples from different parts of the rumen (Pearson \& Smith, 1943; Smith et al. 1956).

Variations roith time of cellulose counts on a practical diet. The method outlined above was applied to the determination over a period of months of the number of 
cellulolytic bacteria $/ \mathrm{ml}$. rumen contents of a sheep receiving a daily ration of $1000 \mathrm{~g}$. chaffed lucerne hay $+16 \mathrm{~g}$. of a balanced mineral lick. On the sampling days, the drinking water was removed from the pen before morning feeding in order to decrease the effect of a variable dilution of the rumen contents with water. The samples were taken $2 \cdot 5 \mathrm{hr}$. after feeding and processed immediately.

The mean values of the counts of cellulolytic colonies in the sixth dilutions are shown in Table 4, the figures being rounded off to the nearest whole number. The coefficient of variation between these results $(\mathbf{7 5 \cdot 2} \%)$ is much higher than that

Table 3. Counts of cellulolytic colonies in five consecutive samples of rumen contents from a sheep fed lucerne hay

Consecutive samples : dilution $1 / 10^{6}$

\begin{tabular}{rrrll}
\hline 1 & 2 & 3 & 4 & 5 \\
18 & 15 & 18 & 27 & 17 \\
20 & 9 & 16 & ${ }^{*}$ & 11 \\
10 & 22 & 19 & 26 & 13 \\
21 & 16 & 19 & 24 & 14 \\
$17 \cdot 25$ & $15 \cdot 5$ & 18 & $25 \cdot 6$ & $13 \cdot 75$
\end{tabular}

$\begin{array}{lc}\text { Mean of } 5 \text { determinations at } 1 / 10^{6} \text { dilution } & 18.03 \\ \text { Standard deviation } & 4.09 \\ \text { Coefficient of variation between consecutive samples } & 22 \cdot 67 \% \\ * \text { Bottle broke. } & \end{array}$

Table 4. Variation of counts of cellulolytic colonies on samples of rumen contents from a sheep fed lucerne hay taken over a period of time

\begin{tabular}{|c|c|c|c|}
\hline & $\begin{array}{l}\text { Mean of colony } \\
\text { counts at dilution }\end{array}$ & & $\begin{array}{l}\text { Mean of colony } \\
\text { counts at dilution }\end{array}$ \\
\hline Date & $1 / 10^{6}$ & Date & $1 / 10^{6}$ \\
\hline 27. iv. 59 & 4 & 2. x. 59 & $\mathbf{3}$ \\
\hline 29 iv. 59 & 6 & 10. xi. 59 & 15 \\
\hline 1. v. 59 & 2 & 11. ii. 60 & 6 \\
\hline 18. vi. 59 & 11 & 13. ii. 60 & 22 \\
\hline 23. vii. 59 & 14 & 18. ii. 60 & 4 \\
\hline \multirow[t]{4}{*}{ 14. viii. 59} & 3 & & \\
\hline & Mean of 11 determinations & $8 \cdot 18$ & \\
\hline & Standard deviation & $6 \cdot 15$ & \\
\hline & Coefficient of variation & $75 \cdot 2 \%$ & \\
\hline
\end{tabular}

found in the case of the five separate samples withdrawn at the same time. From Table 4 it appears that great fluctuations in counts of cellulolytic bacteria did occur within a short time interval. Work is in progress to test whether these fluctuations might have been caused by day-to-day variations in the proportions of fibre to protein in the chaffed lucerne hay.

From the above it may be concluded that the method described in this paper is sufficiently sensitive to measure the fluctuations in the concentrations of the rumen bacteria which ferment the four main carbohydrate constituents of the diet under practical conditions. Compared with the dilution count technique of Wilson \& Briggs (1955), the present method has the disadvantage of being more time-consuming and requiring much more stringent anaerobic precautions. On the other hand, it is much more precise and is capable of showing up smaller variations in the bacterial 
population of the rumen than is the dilution count technique. An additional advantage of our method is that it permits the study of colony shape and the microscopic examination of the colonies from the high dilutions of the sample. This has proved of value in the quantitative study of the change of predominant species within a functional group, following a change of diet. Furthermore, the thinness of the agar film in the roll bottles facilitates the isolation of the predominant species of the different functional groups. Applications of the method to studies on the composition of the bacterial population of the rumen of sheep on two practical diets and during a change from one diet to another will be published elsewhere.

The author wishes to thank the Director of Veterinary Services of the Department of Agriculture for providing the facilities which have made this work possible. Sincere thanks are also due to Dr F. M. C. Gilchrist for her guidance and encouragement, to Mr L. Gouws and Mrs M. Färber for valuable technical assistance and to the Statistics Division of the National Physical Research Laboratory, South African Council for Scientific and Industrial Research, for help with the evaluation of the results.

\section{REFERENCES}

Briggs, C. A. E. (1955). Viable bacteria in the rumen. Dairy Sci. Abstr. 17, 712.

Bryant, M. P. (1959). Bacterial species of the rumen. Bact. Rev. 23, 125.

Bryant, M. P. \& Burkey, L. A. (1953a). Cultural methods and some characteristics of some of the more numerous groups of bacteria in the bovine rumen. J. Dairy Sci. 36, 205.

Bryant, M. P. \& Burkey, L. A. (1953b). Numbers and some predominant groups of bacteria in the rumen of cows fed different rations. J. Dairy Sci. 36, 218.

CooK, A. M. \& STEEL, K. J. (1959). A method for the preparation of bacterial dilutions. Lab. Pract. 8, 359.

Elsden, S. R. \& Philumpon, A. T. (1948). Ruminant digestion. Annu. Rev. Biochem. 17, 705.

Gall, L. S. \& Huhtanen, C. N. (1951). Criteria for judging a true rumen organism and a description of five rumen bacteria. J. Dairy Sci. 34, 353 .

Gilchrist, F. M. C. \& Clark, R. (1957). Refresher courses in physiology. III. The microbiology of the rumen. J.S. Afr. vet. med. Ass. 28, 295.

Huhtanen, C. N. \& Gall, L. S. (1953). Rumen organisms. I. Curved rods and a related rod type. J. Bact. 65, 548.

Huhtanen, C. N., Rogers, M. R. \& GaLx, L. S. (1952). Improved techniques for isolating and purifying rumen organisms. J. Bact. 64, 17.

Hungate, R. E. (1950). The anaerobic mesophilic cellulolytic bacteria. Bact. Rev. 14, 1.

Pearson, R. M. \& Smith, J. A. B. (1943). Utilization of urea in bovine rumen. I. Methods of analysis of rumen ingesta and preliminary experiments in vivo. Biochem. J. 37, 142.

Smith, P. H. \& Hungate, R. E. (1958). Isolation and characterization of Methanobacterium ruminantium n.sp. J. Bact. 75, 713.

Smith, P. H., Sweeney, H. C., Rooney, J. R., King, K. W. \& Moore, W. E. C. (1956). Stratification and kinetic changes in the ingesta of the bovine rumen. J. Dairy Sci. 39, 598.

WiLson, M. K. \& Briggs, C. A. E. (1955). The normal flora of the bovine rumen. II. Quantitative bacteriological studies. J. appl. Bact. 18, 294. 